\title{
Sudden cardiac arrest: Focus on cardiac magnetic resonance
}

\author{
Natalia Ojrzyńska-Witek', Magdalena Marczak², Łukasz Mazurkiewicz'1, Joanna Petryka-Mazurkiewicz², \\ Barbara Miłosz-Wieczorek², Jacek Grzybowski ${ }^{1}$, Mateusz Śpiewak²
}

'Department of Cardiomyopathy, National Institute of Cardiology, Warszawa, Poland

${ }^{2}$ Magnetic Resonance Unit, National Institute of Cardiology, Warszawa, Poland

\author{
Correspondence to: \\ Natalia Ojrzyńska-Witek, MD, \\ $\mathrm{PhD}$, \\ Department of Cardiomyopathy, \\ National Institute of Cardiology, \\ Alpejska 42, 04-628 Warszawa, \\ Poland, \\ phone: +48223434 671, \\ e-mail: nojrzynska@ikard.pl \\ Copyright by the Author(s), 2022 \\ DOI: 10.33963/KP.a2021.0151 \\ Received: \\ September 17, 2021 \\ Accepted: \\ November 2, 2021 \\ Early publication date: \\ November 3, 2021
}

\begin{abstract}
INTRODUCTION
Untreated sudden cardiac arrest (SCA) leads to sudden cardiac death (SCD), which accounts for $15 \%-20 \%$ of all deaths [1]. Cardiovascular diseases, particularly coronary artery disease, have been reported to be the most common cause of SCD [2], except in individuals younger than 30 years, in whom the predominant diagnosis is unexpected, idiopathic cardiac death [3].

Cardiac magnetic resonance (CMR) is suitable for the evaluation of cardiac function and structure, providing deeper insights into these aspects compared with a more widely available echocardiography. This study aimed to investigate the role of CMR in a group of survivors of unexplained SCA.
\end{abstract}

\section{METHODS}

We retrospectively reviewed the medical records (electrocardiography, echocardiography, coronary angiography/coronary artery computed tomography, electrophysiology study) of all patients who underwent CMR between 2008 and 2017 in our hospital. In this study, we enrolled SCA survivors with an initially (before the CMR exam) unexplained or unclear cause of SCA. We included patients with a normal or slightly decreased ejection fraction, as worsening systolic function could be caused by SCA. The study was approved by the local ethics committee. All participants gave written informed consent for the CMR examination.

\section{Cardiac magnetic resonance study}

Cardiac magnetic resonance studies were performed on a 1.5 Tesla scanner (Avanto or Avanto ${ }^{\text {fit }}$, Siemens, Erlangen, Germany) using breath-hold cines in the long-axis view and sequential slices in the short-axis view. All patients underwent a CMR study with a gadolinium-based intravenous contrast agent at standard dosage.

Each study was reported by a team of at least two skilled investigators. The final diagnoses were made using all available data.

\section{Statistical analysis}

Statistical analysis was performed using MedCalc software, version 10.03 (Ostend, Belgium). The Student t-test was used to compare normally distributed variances. A $P$-value $<0.05$ was considered significant. Non-normally distributed data were tested using the Mann-Whitney test. $P<0.05$ was considered significant.

\section{RESULTS AND DISCUSSION}

From all consecutive patients referred for CMR in our hospital between 2008 and $2017(n=8630), 57$ patients were referred due to sudden cardiac arrest. We included 31 patients (16 male) in whom the SCA cause was unclear before CMR. The mean age (standard deviation [SD]) was 36.7 (12.2) years, and the mean (SD) left ventricular ejection fraction was $59.8(7.6) \%$. Late gadolinium enhancement (LGE) was observed in 13 cases (41.9\%), whereas in 1 case, right ventricular LGE was present.

Cardiomyopathies were diagnosed in 3 patients: one patient was found to have dilated cardiomyopathy (DCM), one had left ventricular non-compaction (LVNC), and one had arrhythmogenic right ventricular cardiomyopathy. In patients with DCM and arrhythmogenic right ventricular cardiomyopathy, LGE was observed. All of them had SCA as the first symptom of heart disease, and echocardiography was inconclusive.- 

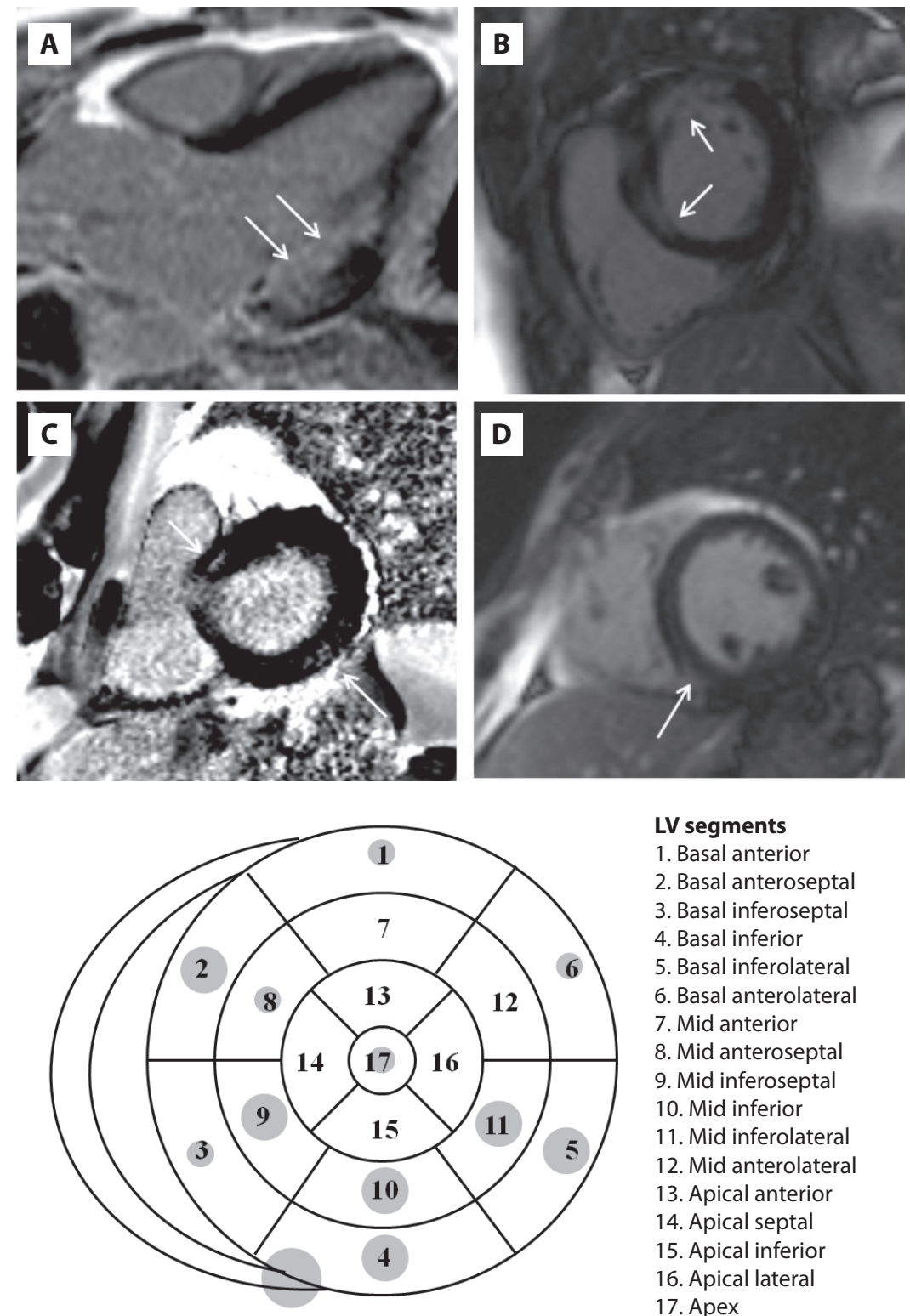

LV segments

1. Basal anterior

2. Basal anteroseptal

3. Basal inferoseptal

4. Basal inferior

5. Basal inferolateral

6. Basal anterolateral

7. Mid anterior

8. Mid anteroseptal

9. Mid inferoseptal

10. Mid inferior

11. Mid inferolateral

12. Mid anterolateral

13. Apical anterior

14. Apical septal

15. Apical inferior

16. Apical lateral

17. Apex
Figure 1. A-D. Examples of LGE found in SCA survivors with normal cardiac function (arrows). A. Three-chamber view of the subendocardial pattern of LGE in a 35-year-old female patient. B. Midventricular short-axis image of the midwall and subendocardial LGE in a 31-yearold male patient. C. Short-axis view of the basal midwall and subepicardial LGE in a 49-year-old male patient. D. Short-axis view of midventricular midwall LGE in a 27-year-old male patient. E. LGE pattern in patients with IVF. Gray dots show localization of fibrosis, and their size corresponds to the number of patients with fibrosis found in specific segments

Abbreviations: IVF, idiopathic ventricular fibrillation; LGE, late gadolinium enhancement; LV, left ventricular; SCA, sudden cardiac arrest
One patient with a normal CMR scan was diagnosed with anorexia and hypokalemia (serum potassium level $<2 \mathrm{mmol} / \mathrm{l})$ as the trigger of SCA. In the remaining 27 (87.1\%) patients, the CMR function was grossly normal, and those patients were rated as having idiopathic ventricular fibrillation (IVF). In 11 of them, a small patch of LGE was seen (Figure 1A-D). Late gadolinium enhancement type, localization, and extension in this group indicated no pattern (Figure 1E). Fibrosis was found in the basal, mid septal, inferior, lateral, and inferior segments, as well as in the apex. Three patients had LGE in the inferior right ventricular insertion point.

There were no statistically significant differences in patients with normal cardiac function with and without fibrosis with regard to age (mean [SD]: 38.8 [12.2] years vs. 37.6 [13.0] years; $P=0.81$ ), left ventricular ejection fraction (mean [SD]: 57.7 [6.8] \% vs. 62.3 [7.0] \%; $P=0.12$ ), left ventricular end-diastolic volumes (median [interquartile range, IQR]: 82.0 [68.7-97.9] $\mathrm{ml} / \mathrm{m}^{2}$ vs 82.5 [76.8-94.4] $\left.\mathrm{ml} / \mathrm{m}^{2} ; P=0.54\right)$, and left ventricular end-systolic volume (mean [SD]: 34.2 [10.3] ml/m² vs. $33.7[11.0] \mathrm{ml} / \mathrm{m}^{2} ; P=0.90$, respectively).

SCA is a major medical problem associated with poor outcomes, with a survival rate of only $9.6 \%$ [4]. In Poland, the out-of-hospital cardiac arrest incidence is 69.7 per 100000 inhabitants [5]. Many cardiovascular diseases are associated with an increased risk of SCA events.

Our cohort consisted of patients with normal or inconclusive echocardiographic findings, whereas in 3 (9.7\%) of them cardiomyopathy was diagnosed by CMR. In the rest, mechanical cardiac function was grossly normal. In a large cohort of patients with normal echocardiography and ventricular arrhythmias (including aborted SCA) [6], structural heart disease was found in $25.5 \%$ of patients. 
In patients with idiopathic DCM, approximately $30 \%$ of deaths are sudden [7] and strongly correlated with the presence of LGE [8]. LVNC is associated with a higher risk of SCD and ventricular arrhythmias than age-matched DCM [9]. Arrhythmias in patients with LVNC occur even if LGE is not present [10]. Accordingly, in patients with arrhythmogenic right ventricular cardiomyopathy, life-threatening arrhythmias can occur before detection of histological and imaging features [11].

In $87.1 \%$ of patients with normal cardiac function, there were no other causes according to a previous (pre-CMR) investigation. The CMR examination led to the finding of a potential arrhythmic substrate (represented by LGE) that might cause VT/VF leading to SCA in 11 of 27 patients with IVF.

In multiple studies analyzing causes of SCD in the US and European countries (Italy, Denmark, and the United Kingdom), the most common cause was unexplained SCD in patients with structurally normal hearts [12]. In the out-of-hospital cardiac arrest real-life cohort registry, IVF was diagnosed in $6.8 \%$ of SCAs among all survivors of SCAs with cardiac etiologies [13].

SCA survivors with CMR-based diagnosis have been found to have a worse prognosis than patients with a normal scan or minor nonspecific changes [14]. Moreover, the presence and extent of LGE correlate with a worse prognosis [15].

\section{Limitation and strength}

To our knowledge, this is the first study evaluating the CMR values in survivors of SCA of unknown etiology in a Polish population. This study was conducted within the context of a limited cohort and retrospective analyses. Patients were referred for CMR study by a primary physician. We retrospectively recruited patients after SCA with good outcomes. Consequently, we examined only SCA survivors who did not represent the whole population with SCA. This could explain why our cohort consisted mostly of young people without comorbidities and why IVF was so frequent.

\section{CONCLUSION}

In a cohort of unexplained SCA survivors, the CMR study led to the finding of underlying cardiomyopathy in 3 patients (9.7\%) and of potential arrhythmic substrates in 11 (35.5\%) patients with idiopathic ventricular fibrillation. In 16 (51.6\%) patients with IVF, the CMR scan was normal. The role of CMR in SCA survivors needs further investigation.

\section{Article information}

Conflict of interest: None declared.

Open access: This article is available in open access under Creative Common Attribution-Non-Commercial-No Derivatives 4.0 International (CC BY-NC-ND 4.0) license, allowing to download articles and share them with others as long as they credit the authors and the publisher, but without permission to change them in any way or use them commercially. For commercial use, please contact the journal office at kardiologiapolska@ptkardio.pl.

\section{REFERENCES}

1. Hayashi M, Shimizu W, Albert CM. The spectrum of epidemiology underlying sudden cardiac death. Circ Res. 2015; 116(12): 1887-1906, doi: 10.1161/CIRCRESAHA.116.304521, indexed in Pubmed: 26044246.

2. Adabag AS, Peterson G, Apple FS, et al. Etiology of sudden death in the community: results of anatomical, metabolic, and genetic evaluation. Am Heart J. 2010; 159(1): 33-39, doi: 10.1016/j.ahj.2009.10.019, indexed in Pubmed: 20102864.

3. Bagnall RD, Weintraub RG, Ingles J, et al. A prospective study of sudden cardiac death among children and young adults. N Engl J Med. 2016; 374(25): 2441-2452, doi: 10.1056/NEJMoa1510687, indexed in Pubmed: 27332903.

4. McNally B, Robb R, Mehta M, et al. Centers for Disease Control and Prevention. Out-of-hospital cardiac arrest surveillance - Cardiac Arrest Registry to Enhance Survival (CARES), United States, October 1, 2005-December 31, 2010. MMWR Surveill Summ. 2011; 60(8): 1-19, indexed in Pubmed: 21796098.

5. Nadolny K, Zyśko D, Obremska M, et al. Analysis of out-of-hospital cardiac arrest in Poland in a 1-year period: data from the POL-OHCA registry. Kardiol Pol. 2020; 78(5): 404-411, doi: 10.33963/KP.15241, indexed in Pubmed: 32191020.

6. Andreini D, Dello Russo A, Pontone G, et al. CMR for identifying the substrate of ventricular arrhythmia in patients with normal echocardiography. JACC Cardiovasc Imaging. 2020; 13(2): 410-421, doi: 10.1016/j. jcmg.2019.04.023, indexed in Pubmed: 31326488.

7. Tamburro P, Wilber D. Sudden death in idiopathic dilated cardiomyopathy. Am Heart J. 1992; 124(4): 1035-1045, doi: 10.1016/0002-8703(92)90989-9, indexed in Pubmed: 1529877.

8. Di Marco A, Anguera I, Schmitt M, et al. Late gadolinium enhancement and the Risk for ventricular arrhythmias or sudden death in dilated cardiomyopathy: systematic review and meta-analysis. JACC Heart Fail. 2017; 5(1): 28-38, doi: 10.1016/j.jchf.2016.09.017, indexed in Pubmed: 28017348.

9. Sedaghat-Hamedani F, Haas J, Zhu F, et al. Clinical genetics and outcome of left ventricular non-compaction cardiomyopathy. Eur Heart J. 2017; 38(46): 3449-3460, doi: 10.1093/eurheartj/ehx545, indexed in Pubmed: 29029073.

10. Nucifora G, Aquaro GD, Pingitore A, et al. Myocardial fibrosis in isolated left ventricular non-compaction and its relation to disease severity. Eur J Heart Fail. 2011; 13(2): 170-176, doi: 10.1093/eurjhf/hfq222, indexed in Pubmed: 21208941.

11. Marcus Fl, McKenna WJ, Sherrill D, et al. Diagnosis of arrhythmogenic right ventricular cardiomyopathy/dysplasia: proposed modification of the task force criteria. Circulation. 2010; 121(13): 1533-1541, doi: 10.1161/CIRCULATIONAHA.108.840827, indexed in Pubmed: 20172911.

12. Asif IM, Harmon KG. Incidence and etiology of sudden cardiac death: new updates for athletic departments. Sports Health. 2017; 9(3): 268-279, doi: 10.1177/1941738117694153, indexed in Pubmed: 28452637.

13. Waldmann V, Bougouin W, Karam N, et al. Paris-SDEC investigators. Characteristics and clinical assessment of unexplained sudden cardiac arrest in the real-world setting: focus on idiopathic ventricular fibrillation. Eur Heart J. 2018;39(21): 1981-1987, doi: 10.1093/eurheartj/ehy098, indexed in Pubmed: 29566157.

14. Rodrigues $P$, Joshi $A$, Williams $H$, et al. Diagnosis and prognosis in sudden cardiac arrest survivors without coronary artery disease. Circ Cardiovasc Imaging. 2017; 10(12): e006709, doi: 10.1161/circimaging.117.006709, indexed in Pubmed: 29237609.

15. Neilan TG, Farhad H, Mayrhofer T, et al. Late gadolinium enhancement among survivors of sudden cardiac arrest. JACC Cardiovasc Imaging. 2015; 8(4):414-423, doi: 10.1016/j.jcmg.2014.11.017, indexed in Pubmed: 25797123. 